\section{SB R SMALL BUSINESS INTERNATIONAL REVIEW}

Volumen 3, Número 1

Enero - junio 2019

pp. 36-48

DOI: https://doi.org/10.26784/sbir.v3il.172 E-ISSN: 2531-0046

\title{
Análisis de las relaciones entre los tipos de innovaciones y el desempeño empresarial en parques tecnológicos
}

\section{Analysis of relations between the types of innovations and performance in technological parks}

Lucas Bonacina Roldan

Profesor, Escuela de Negocios, Pontifícia Universidade Católica do Rio Grande do Su, Brasil. Doctor en Administración.

E-mail: lucas.roldan@pucrs.br

ORCID: http://orcid.org/0000-0002-3881-3918

William Neves Bastos

Graduado en Administración, Pontifícia Universidade Católica do Rio Grande do Sul, Brasil

E-mail: william.neves.bastos@gmail.com

ORCID: http://orcid.org/0000-0001-5261-0597

\section{Resumen}

En la actualidad es indiscutible la necesidad de las empresas de estar siempre innovando para mantenerse competitivas. Sin embargo, el impacto de estas innovaciones entre ellas y en el propio desempeño de la empresa necesita ser mejor explotado y comprendido. El presente artículo analiza la relación entre los tipos innovaciones y el desempeño en los parques tecnológicos brasileños. Para alcanzar el objetivo, se realizó una survey con 231 empresas residentes en los parques tecnológicos brasileños y el análisis de los datos obtenidos se dio a través del modelado de ecuaciones estructurales. Como resultados, se ha identificado que las innovaciones organizacionales influencian las innovaciones de proceso y de marketing, las innovaciones de proceso influencian la innovación de producto y el desempeño organizacional y la innovación de marketing influye en el desempeño organizacional.

Palabras-clave: innovación, parques tecnológicos, desempeño. 


\begin{abstract}
The need for companies to always innovate to stay competitive makes them invest in innovations. The impact of these innovations between them and the performance of innovations needs to be better explored and understood. This article analyzes the relationship between innovation types and performance in Brazilian technology parks. To reach the objective, a survey was conducted with 231 companies resident in the Brazilian technology parks and the analysis of the data obtained was done through the modeling of structural equations. As a result, it was identified that organizational innovations influence process and marketing innovations, process innovations influence product innovation and organizational performance, and marketing innovation influences organizational performance.
\end{abstract}

Keywords: innovation, technology parks, performance. 


\section{Introducción}

La innovación es una oportunidad para que las empresas emprendedoras generen ingresos mediante la creación de un monopolio temporal y la innovación continua es considerada la principal fuente de éxito empresarial a largo plazo (Schumpeter, 1939). Las empresas necesitan ser innovadoras, para que así puedan perpetuarse en un entorno competitivo (Hult, Hurley, \& Knight, 2004)we address three research questions: (1. Los beneficios de las empresas innovadoras no se limitan apenas a ellas, ya que movilizan su región y su país, posibilitando el aumento del nivel de empleo y renta (Geldes, Felzensztein, \& Palacios-Fenech, 2017). Considerando que las innovaciones permiten que las empresas puedan aumentar sus desempeños accediendo a nuevos mercados, adquiriendo nuevos conocimientos y aumentando el valor de sus marcas, por lo que es importante para la sustentabilidad de las empresas (Crossan \& Apaydin, 2010).

La forma más recurrente para intentar cuantificar los resultados de las empresas es el rendimiento financiero (Madrid-Guijarro, García-Pérez-de-Lema, \& Van Auken, 2013). No obstante, aunque él acople resultados como lucro y rentabilidad, él sólo no dimensiona todos los tipos de innovación, así existe la necesidad de utilizar otras dimensiones para medir el impacto de las innovaciones en las empresas (Gunday, Ulusoy, Kilic, \& Alpkan, 2011). Las empresas tienen la posibilidad de innovar en diversas áreas a lo largo del tiempo, pudiendo así tener innovaciones en productos, en proceso, en marketing (OECD, 2007). Y en su forma de organización que influyen en su desempeño (Gunday et al., 2011, Hassan, Shaukat, Nawaz, \& Naz, 2013).

Un ambiente que tiene como objetivo el fomento a la innovación son los parques tecnológicos y las incubadoras (Díeez-Vial, Fernández-Olmos, Díez-Vial, \& Fernández-Olmos, 2015; Gkypali, Kokkinos, Bouras, \& Tsekouras, 2016; Soetanto \& Jack, 2016). Cuando las empresas se localizan en un entorno de innovación, esperan más que un lugar apropiado para sus actividades fines, pero un ambiente propicio al network y un flujo de conocimiento que pueda desarrollar innovaciones y posteriormente dar algún tipo de resultado (Gunday et al., 2011; Hassan et al., 2013;
Roldan, 2017; Ulusoy, Gunday, Alpkan, \& Kilic, 2008). La relación entre los tipos de innovación y el desempeño es objetivo recurrente en la literatura internacional (Geldes et al., 2017; Gunday et al., 2011; Karabulut, 2015). Sin embargo, es necesario explorar mejor estas relaciones en los ambientes de innovación ya que este trabajo posee como unidad de análisis a las empresas residentes en los parques tecnológicos brasileños, los cuales pueden prestar servicio de apoyo.

El problema del estudio de este artículo es ¿cuál es la relación entre los tipos de innovación y el desempeño en las empresas residentes en parques tecnológicos brasileños? Por lo tanto tiene como objetivo analizar las relaciones entre los tipos de innovaciones y el desempeño en empresas residentes en los parques tecnológicos brasileños. Así, el desarrollo del mismo es el siguiente: luego de la introducción se encuentra una revisión de la literatura con conceptos y características de innovación y desempeño, además de la estructuración de las hipótesis. Después se encuentra la metodología utilizada. Seguidamente se presenta el análisis y discusión de los resultados y, finalmente, la conclusión de la investigación con la limitación y sugerencias para temas de nuevas investigaciones.

\section{Revisión de la literatura e hipótesis}

Las empresas necesitan ser más competitivas en sus mercados de actuaciones, debido a la fuerte competencia a nivel global, desarrollan habilidades emprendedoras, para así poder aplicarlas y ser exitosas (Karabulut, 2015, Karlsson \& Tavassoli, 2016). Las innovaciones tienen gran importancia para las empresas, puesto que poseen potencial de aumentar la eficiencia y la rentabilidad de las mismas (Soetanto \& Jack, 2013).

La innovación es la transformación de conocimiento técnico en algún valor comercial, pueden ser de cuatro tipos: de producto, de proceso, de marketing y organizacional (OECD, 2007). Existen diferencias entre los niveles de capacidad de innovar, sin embargo, existe una posibilidad de interacción entre los tipos de innovación; esto puesto, tiene muchos aspectos que deben ser enfocados al mismo tiempo 
en las actividades innovadoras, como nuevas prácticas organizacionales, nuevas prácticas de marketing, nuevos productos, nuevos sistemas administrativos y nuevas tecnologías de procesos (Azar \& Ciabuschi, 2017; Gunday et al., 2011; Ulusoy et al., 2008).

La innovación de producto (IPROD) corresponde a la introducción de un bien o servicio nuevo o significativamente mejorado. Se incluyen mejoras significativas en especificaciones técnicas, componentes y materiales, software incorporado, facilidad de uso u otras características funcionales. En cuanto a las innovaciones organizativas (IORG) es la implementación de un nuevo método organizativo en las prácticas de negocios de la empresa, en la organización de su lugar de trabajo o en sus relaciones externas. La innovación de proceso (IPROC) es la implementación de un método de producción o distribución nuevo o significativamente mejorado. Se incluyen cambios significativos en técnicas, equipos y / o software. La innovación de marketing (IMKT) es la implementación de un nuevo método de marketing con cambios significativos en el diseño del producto o en su embalaje, en el posicionamiento del producto, en su promoción o en la fijación de precios (OECD, 2007).

Las innovaciones organizacionales, o renovaciones organizacionales que traen mejoras estructurales y que pueden llevar a una mejor coordinación organizacional y los mecanismos de cooperación pueden contribuir a un ambiente más propicio para otros tipos de innovaciones (producto, proceso y marketing) (Gunday et al., 2011). La innovación organizacional es la base tanto para las innovaciones de marketing como para los procesos para impactar la innovación del producto (Ulusoy et al., 2008). Para los países menos desarrollados, la innovación organizacional debe ser abordada como un principal factor determinante (Zuñiga-Collazos, 2018). Un estudio hecho con empresas de economías emergentes de Colombia, Perú y Chile muestra la relación positiva y significativa entre innovación organizacional (IORG) y la innovación de marketing (IMKT) indicando que la IORG es la base para otros tipos de innovaciones dentro de las empresas (Pino, Felzensztein, Zwerg-villegas, \& Arias-bolzmann, 2016). En contraposición a un estudio en la industria manufacturera en Corea del Sur, no hay una relación causal entre innovación organizacional e innovación de productos, por otro lado, existe una fuerte relación causal entre innovación organizacional e innovación de proceso. Además, hay una relación causal entre innovación organizacional e innovación de marketing. Es decir, las empresas deben considerar el papel de la innovación organizacional cuando realizan actividades de innovación. (Chung \& Kim, 2017). Por lo tanto, la hipótesis 1 de esta investigación se presenta a continuación:

Hipótesis 1. Existe una relación positiva entre la innovación organizacional y otros tipos de innovación.

H1a. Cuanto mayor sea el nivel de innovación organización, mayor será el nivel de innovación de producto.

H1b. Cuanto mayor sea el nivel de innovación organizacional, mayor será el nivel de innovación de proceso.

H1c. Cuanto mayor sea el nivel de innovación organizacional, mayor será el nivel de innovación de marketing.

El estudio dirigido por Gunday (2011) con industrias en Turquía mostró que la innovación de proceso influye positivamente en un mayor nivel de innovaciones de productos, soluciones innovadoras que proporcionan las etapas de los procesos de producción con ventajas recién mejoradas como calidad de producción, valor, la velocidad y el bajo costo pueden aumentar las posibilidades de los nuevos componentes, ingredientes, especificaciones técnicas, funcionalidades entre otras, para el producto satisfacer mejor las necesidades y los deseos de los clientes en un nivel, pero en un contexto diferente estudiado en el presente artículo. En Indonesia, que investigó el sector de mármol y artesanía, afirmó que la innovación del proceso tiene un efecto positivo significativo en la innovación de productos (Astuti, Prawoto, Irawan, \& Sugiono, 2018). El efecto del proceso de innovación de marketing es a través de las innovaciones de productos (Ulusoy et al., 2008). Es así que se forma la Hipótesis 2 presentada abajo:

Hipótesis 2. Cuanto mayor sea el nivel de innovación de proceso, mayor nivel de innovación de productos. 
En el contexto latinoamericano, las empresas tienen recursos limitados, menores tasas de inversión en $P \& D$ y derechos de propiedad intelectual más elevados, por lo que las innovaciones no tecnológicas (marketing y organizacional) representan una manera estratégica de innovar y mejorar el desempeño del mercado, mercado en las empresas exportadoras de las economías emergentes de América del Sur (Pino et al., 2016). Los resultados de una investigación realizada soportan que las innovaciones de marketing afectan positivamente el desempeño de la producción (Ulusoy et al., 2008). En las empresas de fabricación en Turquía IMKT tuvieron resultados positivos y significativos con IPROD (Gunday et al., 2011). Una estrategia de innovación de marketing tiene una relación positiva con la innovación de productos (Aksoy, 2017). Un estudio realizado en empresas pequeñas y medianas del sector minorista en España afirma que la introducción de nuevos métodos de marketing tiene un impacto positivo en la propensión a realizar todos los demás tipos de innovación (producto, proceso y organización) (Medrano, \& Olate-Pascual, 2016). Así emerge la Hipótesis 3 presentada a continuación:

Hipótesis 3. Cuanto mayor sea el nivel de innovación de marketing, mayor será el nivel de innovación.

H3a. Cuanto mayor sea el nivel de innovación de marketing, mayor será el nivel de innovación de producto.

H3b. Cuanto mayor sea el nivel de innovación de marketing, mayor será el nivel de innovación de proceso.

Este estudio tiene como objetivo entender las relaciones entre los tipos de innovación y los tipos de desempeño que una empresa innovadora puede tener (DO), en un estudio dirigido por Gunday (2011), revela que el rendimiento financiero es un producto de actuación innovadora, productiva y de mercado, aún en el mismo estudio se debe tener en cuenta que las empresas innovadoras tienen mayor participación de mercado, ventas totales y exportaciones, se afirma que los cuatro tipos de innovaciones desempeñan un papel positivo y significativo (Gunday et al., 2011). Mientras más un individuo u organización demuestra una comprensión más completa de la innovación, hay mayor propensión para alcanzar resultados (Kahn, 2018). Las innovaciones no tecnológicas (organizacional y marketing) son maneras de las empresas de innovar y aumentar sus divisiones de mercado (Pino et al., 2016). La innovación de productos tiene el significativo efecto sobre el desempeño innovador (Astuti et al., 2018). La IMKT tiene una relación positiva con el rendimiento y que IPROD tiene una relación positiva y significativa con el desempeño del mercado (Aksoy, 2017). La implementación de innovación de marketing aplicada aparentemente no logró aumentar el desempeño (Astuti et al., 2018). Si desea mejorar el rendimiento innovador, las empresas deben centrarse en las actividades de innovación de procesos, marketing y organización, en lugar de las actividades de innovación de productos (Tuan, Nhan, Giang, \& Ngoc, 2016). De esta forma son presentadas las Hipótesis 4, 5 y 6.

Hipótesis 4. Cuanto mayor sea el nivel de innovación de producto, mayor será el desempeño organizacional.

Hipótesis 5. Cuanto mayor sea el nivel de innovación de proceso, mayor será el desempeño organizacional.

Hipótesis 6. Cuanto mayor sea el nivel de innovación de marketing, mayor será el desempeño organizacional.

A continuación se presenta en la Figura 1 el modelo teórico de este estudio.

Figura 1. Modelo Teórico

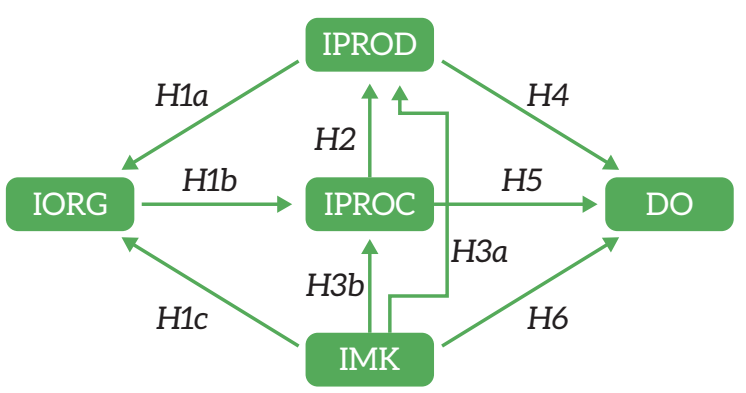

Fuente: elaboración propia.

\section{Metodología}

\subsection{Población y muestra}

Para alcanzar el objetivo propuesto se realizó una investigación cuantitativa de cuño descriptivo, para un mejor entendimiento de cómo los tipos de innovaciones pueden llevar a desempeños superiores. 
La recolección de los datos se realizó en 2016 entre los meses de enero y junio. El instrumento de recolección fue compuesto de 29 ítems siendo divididos en 5 dimensiones. Después de la elaboración del instrumento de recolección de datos, se realizaron entrevistas con tres investigadores, seis gestores del Parque Científico y Tecnológico de la PUCRS (TECNOPUC) y cinco empresas residentes en el parque con el objetivo de ajustar a la realidad de las empresas. Después, a los pequeños ajustes sugeridos fueron realizados y se aplicó un pre-test con (34 empresas).

El cuestionario estructurado de auto llenado fue encaminado a 1064 empresas residentes en parques tecnológicos brasileños, habiendo sido obtenida una muestra de doscientos treinta y una ( $\mathrm{n}=$ 231) respuestas válidas de los gestores de las empresas residentes en los 22 parques tecnológicos brasileños (considerados consolidados) en operación en Brasil, (parques con empresas residentes en ellos hace por lo mínimo un año), distribuidos en las diversas regiones del país y vinculados a la ANPROTEC (Associação Nacional de Entidades Promotoras de Empreendimentos Inovadores), que ayudó en la distribución del cuestionario, apoyando y enviando la investigación por e. -mail a todos sus asociados.

Fue criterio para la participación del estudio, la empresa residir por lo menos un año en el parque, las empresas se clasificaron por su tamaño, basándose en el número de empleados, de acuerdo con la clasificación adoptada por el SEBRAE (Serviço Brasileiro de Apoio às Micro e Pequenas Empresas), que asegure criterios del IBGE (Instituto Brasileiro de Geografia e Estatística) para comercio y servicios (SEBRAE).

En la búsqueda de cortar las desviaciones perjudiciales (casos omisos y outliers) a la aplicación de las técnicas estadísticas de análisis del modelo teórico, se aplicaron filtros de purificación al banco de datos. Como resultado, en esta etapa se eliminaron 42 cuestionarios. Después de la purificación de los datos, con base en la muestra válida $(n=189)$ compuesta por empresas residentes en 22 parques tecnológicos, esparcidos en 12 estados brasileños.

\subsection{Medición de variables y análisis estadísticos}

Para ejecutar la prueba del modelo teórico propuesto, se utilizaron escalas establecidas y probadas en otras investigaciones (Gunday et al., 2011). La escala sobre innovaciones de producto / servicios tenía como objetivo medir la frecuencia de la misma (1nunca y 5- muy frecuente), las escalas utilizadas para medir las innovaciones de procesos en las empresas en los últimos tres años (1-nunca y 5- muy frecuente), para medir las innovaciones de marketing en su empresa en los últimos tres años se usó la escala (1-nunca y 5- muy frecuente), la medición de innovaciones organizacionales en la empresa en los últimos tres años (1- nunca y 5 - muy frecuente) y la medición del desempeño organizacional tuvo como objetivo analizar la posición competitiva de su empresa durante los últimos tres años en relación a ella misma considerando una escala donde (1- bajo desempeño durante los últimos tres años y 5- desempeño alto durante los últimos tres años).

Los constructos teóricos fueron preparadosa través del análisis descriptivo de las variables observadas. Después de la operacionalización de los constructos, se realizó un análisis factorial exploratorio (AFE) para verificar si las suposiciones del análisis factorial eran atendidas por cada una de las escalas de medición de los principales constructos.

La técnica de modelado de ecuaciones estructurales (MEE) fue elegida para evaluar las hipótesis desarrolladas, adoptando la técnica multivariada. Para evitar las consecuencias de algunas deducciones precipitadas, se adoptó el enfoque Two-step, aplicando los procesos de Análisis Factorial Confirmatorio (AFC), para demostrar las relaciones de las medidas observadas en el constructo y el modelo de medición verificando las reglas de Confiabilidad Compuesta (AFC) (CC), de Análisis de Variancia Media Extraída (AVE), de Validez Convergente (VC) y de Validez Discriminante(VD), para analizar su validez. Después del análisis del modelo de medición, se analizó el modelo estructural para que entre las posibles relaciones de dependencia, fueran mostradas las asociaciones entre los constructos del modelo y, para su validación, utilizando el AMOS $®$, se consideró satisfactoria la relación cuando se especifica el nivel de significancia en $\mathrm{p} \leq 0,05$ (Hair, Black, Babin, Anderson, \& Tatham, 2009). 


\section{Resultados}

Para caracterizar mejor las empresas analizadas, se analizaron algunas variables de control, siendo donde el 41,8\% eran empresas incubadas, el 30,2\% no eran incubadas y el $27,5 \%$ ya no eran más incubadas, pero ya pasaron por este proceso y hoy están residiendo en parques tecnológicos. En cuanto a la edad de las empresas, la media permeó los 7,9 años (Desviación estándar $=8,142$ ). En cuanto al tamaño de las empresas, fue medido a partir de la cantidad de empleados de la misma de acuerdo con el Serviço Brasileiro de Apoio às Micro e Pequenas Empresas (SEBRAE), siendo que el 61,1\% son microempresas, el 27,6\% pequeño tamaño, 8,6\% de gran tamaño y $2,7 \%$ de tamaño mediano.

La dimensión de Innovación de Producto presenta una tendencia de mayor ocurrencia, ya que presentó medias entre 3,54 y 4,27 con pequeñas desviaciones estándar, lo que está de acuerdo con los resultados de la etapa cualitativa. Estas medias fueron más altas que las encontradas en el estudio de Gunday et al. (2011).

En el análisis de la dimensión Innovación de Proceso también presenta una media por encima de la neutralidad, pero un poco por debajo de la dimensión anterior, ya que las medias se encuentran entre 3,6 y 3,93. Tales medias también fueron superiores a las encontradas en el estudio de Gunday et al. (2011).

En cuanto a la dimensión Innovación de Marketing, las medias también fueron un poco por encima de la centralidad con la media entre 3,01 y 3,58. Como ocurrió en las demás innovaciones ya mencionadas, este resultado también fue mayor que el encontrado por Gunday et al. (2011), reforzando que el ambiente de los parques debe favorecer la ocurrencia de innovaciones en las empresas o estas empresas posean características más innovadoras.

En la dimensión de Innovación Organizacional las medias se ubicaron entre 3,06 y 3,78 y estos resultados también fueron mayores que el estudio de Gunday et al. (2011). Por último, la dimensión Desempeño Organizacional presentó un desempeño intermedio ligeramente inclinado hacia arriba con medias entre 3,10 y 3,30. El resultado del desempeño fue similar al encontrado en el estudio de Gunday et al. (2011).
A través de los resultados presentados, se percibe que el ambiente brasileño de los parques tecnológicos aún puede ser perfeccionado, ya que las empresas todavía no utilizan con mucha frecuencia los beneficios que los parques ofrecen para el fomento a la innovación, pues los promedios todavía pueden ser mayores de acuerdo con los entrevistados. La ocurrencia de las innovaciones (producto, proceso, organización y marketing) comparado con el estudio que fue utilizado como base para la adaptación de las escalas fue mayor, demostrando que en el contexto de los parques tecnológicos brasileños ocurren proactivamente más innovaciones que en las industrias de Turquía. En cuanto al rendimiento financiero de las empresas, los resultados fueron similares al contexto del trabajo de Gunday et al., (2011).

Para extraer las relaciones presentadas en las hipótesis, se realizó un análisis multivariado en dos fases. En la primera etapa se realizó un análisis factorial de la estructura de investigación a través de un análisis de componentes principales (ACP) para reducir el conjunto de variables con el objetivo de trabajar mejor las escalas, una vez que el número inicial de variables era muy grande para conducir un análisis de conexiones individuales.

Se realizó una ACP con rotación Varimax para descubrir las dimensiones subyacentes entre los constructos analizados. En esta etapa poseyó el objetivo de explorar la consistencia interna y la confiabilidad (validez de contenido) entre los ítems de cada constructo a través del Alpha de Cronbach y pruebas de unidimensionalidad. Además, la validez discriminante entre las construcciones de innovación también es examinada y verificada por la prueba de varianza media extraída (AVE). La segunda etapa involucró el análisis de las relaciones entre estos factores usando el enfoque de modelado de ecuaciones estructurales (MEE).

Para fines de análisis de los modelos de medición de los constructos se realizó una separación (Hair et al., 2009). Así, se realizó un Análisis Factorial Exploratorio de las relaciones de los tipos de innovaciones y el desempeño. En la Tabla 1 abajo se presenta el análisis de los componentes principales de las innovaciones y del desempeño. 
Tabla 1. Análisis de los Componentes Principales de las Innovaciones y del Desempeño

\begin{tabular}{|c|c|c|c|c|c|}
\hline Factores & $\begin{array}{l}\text { Cargas } \\
\text { Factoriales }\end{array}$ & $\begin{array}{c}\% \\
\text { Variancia } \\
\text { Explicada }\end{array}$ & $\begin{array}{c}\text { ade } \\
\text { Cronbach }\end{array}$ & CC & AVE \\
\hline Factor 1: Innovaciones de Producto/Servicio & & 53,561 & ,818 & ,871 & ,638 \\
\hline $\begin{array}{l}\text { Desarrollo de nuevos productos y / o servicios con } \\
\text { las especificaciones técnicas y funcionalidades } \\
\text { totalmente diferentes de las actuales }\end{array}$ & 698 & & & & \\
\hline $\begin{array}{l}\text { Desarrollo de novedades para los productos y / o } \\
\text { servicios actuales con miras a mejorar la facilidad } \\
\text { de uso para los clientes }\end{array}$ & 794 & & & & \\
\hline $\begin{array}{l}\text { Desarrollo de novedades para los productos y / o } \\
\text { servicios actuales con miras a mejorar la } \\
\text { satisfacción de los clientes }\end{array}$ & 801 & & & & \\
\hline $\begin{array}{l}\text { Desarrollo de productos y / o servicios con nuevos } \\
\text { componentes y materiales totalmente diferentes } \\
\text { de los actuales. }\end{array}$ & ,737 & & & & \\
\hline Factor 2: Innovaciones de Proceso & & 62,077 & ,847 & ,905 & 658 \\
\hline $\begin{array}{l}\text { Eliminación de actividades sin valor agregado en } \\
\text { procesos }\end{array}$ & ,721 & & & & \\
\hline $\begin{array}{l}\text { Diminuiç̧ão de custos em processos de manufatura, } \\
\text { serviços, técnicas, maquinário ou desenvolvimento } \\
\text { de software. }\end{array}$ & 813 & & & & \\
\hline $\begin{array}{l}\text { Aumento de la calidad de los procesos de } \\
\text { manufactura, servicios, técnicos, maquinaria o } \\
\text { desarrollo de software. }\end{array}$ & ,774 & & & & \\
\hline $\begin{array}{l}\text { Reducción de costos en actividades sin valor } \\
\text { agregado en procesos de entrega }\end{array}$ & ,823 & & & & \\
\hline $\begin{array}{l}\text { Disminución de costos y / o aumento en la } \\
\text { velocidad de entrega en procesos logísticos }\end{array}$ & 804 & & & & \\
\hline Factor 3: Innovaciones de Marketing & & 56,354 & 800 & ,831 &, 556 \\
\hline $\begin{array}{l}\text { Cambio del diseño de los productos y / o servicios } \\
\text { actuales y / o nuevos por medio de cambios, como } \\
\text { en la apariencia, embalaje, forma y volumen sin } \\
\text { alterar sus características técnicas y funcionales } \\
\text { básicas }\end{array}$ & ,640 & & & & \\
\hline $\begin{array}{l}\text { Cambio de los canales de distribución, sin alterar } \\
\text { los procesos logísticos relacionados con la entrega } \\
\text { del producto y / o servicio }\end{array}$ & ,777 & & & & \\
\hline $\begin{array}{l}\text { Cambio de las técnicas de promoción de productos } \\
\text { y / o servicios utilizados para la promoción de los } \\
\text { productos actuales y / o nuevos }\end{array}$ & ,832 & & & & \\
\hline $\begin{array}{l}\text { Cambio de las técnicas de precios de los productos y } \\
\text { / o servicios para la fijación del precio de los } \\
\text { productos actuales y / o nuevos }\end{array}$ & ,671 & & & & \\
\hline Cambio de las actividades de gestión de marketing & 812 & & & & \\
\hline
\end{tabular}


Tabla 1. Análisis de los Componentes Principales de las Innovaciones y del Desempeño (continuación)

\begin{tabular}{|c|c|c|c|c|c|}
\hline Factores & $\begin{array}{l}\text { Cargas } \\
\text { Factoriales }\end{array}$ & $\begin{array}{l}\% \\
\text { Variancia } \\
\text { Explicada }\end{array}$ & $\begin{array}{c}\text { ade } \\
\text { Cronbach }\end{array}$ & $\mathrm{CC}$ & AVE \\
\hline Factor 4: Innovaciones Organizacionales & & 48,397 & ,864 & ,911 & ,598 \\
\hline $\begin{array}{l}\text { Cambio en la estructura organizacional de rutinas, } \\
\text { procedimientos y procesos empleados para realizar } \\
\text { actividades }\end{array}$ & ,702 & & & & \\
\hline $\begin{array}{l}\text { Cambio en la estructura organizacional del sistema } \\
\text { de gestión de la cadena de suministro }\end{array}$ & ,705 & & & & \\
\hline $\begin{array}{l}\text { Cambio en la estructura organizacional de los } \\
\text { sistemas de gestión de producción y calidad }\end{array}$ & ,661 & & & & \\
\hline $\begin{array}{l}\text { Cambio en la estructura organizacional del sistema } \\
\text { de gestión de recursos humanos }\end{array}$ & 684 & & & & \\
\hline $\begin{array}{l}\text { Cambio en la estructura organizacional del sistema } \\
\text { de gestión de la información y el intercambio de } \\
\text { información }\end{array}$ & 663 & & & & \\
\hline $\begin{array}{l}\text { Cambio en la estructura organizacional para } \\
\text { facilitar el trabajo en equipo }\end{array}$ & ,719 & & & & \\
\hline $\begin{array}{l}\text { Cambio en la estructura organizacional para } \\
\text { facilitar la coordinación entre diferentes funciones, } \\
\text { tales como marketing, gestión de personas y } \\
\text { desarrollo de producto y / o servicio }\end{array}$ & ,760 & & & & \\
\hline $\begin{array}{l}\text { Cambio en la estructura organizacional para } \\
\text { facilitar la organización de acuerdo con el tipo de } \\
\text { proyecto a ser realizado }\end{array}$ & ,741 & & & & \\
\hline $\begin{array}{l}\text { Cambio en la estructura organizacional de la } \\
\text { estructura organizacional para facilitar } \\
\text { asociaciones estratégicas y colaboraciones de } \\
\text { negocios a largo plazo en la empresa }\end{array}$ & ,614 & & & & \\
\hline Factor 5: Desempeño Organizacional & & 71,862 & ,902 & ,943 & ,770 \\
\hline Retorno sobre activos (lucro / total del activo) & ,868 & & & & \\
\hline Rentabilidad general de la empresa & 891 & & & & \\
\hline Retorno sobre las ventas (lucro / ventas totales) & 870 & & & & \\
\hline Flujo de caja excluyendo inversiones & ,803 & & & & \\
\hline Ventas totales & ,801 & & & & \\
\hline
\end{tabular}

KMO Medida de la adecuación de la muestra = 0.854 Prueba de esfericidad de Bartlett $=3480,607 ; \mathrm{p}<0.000$

Fuente: Elaboración propia en base a los datos de la investigación. 
En el análisis de los resultados de la Tabla 1, se verifica que los factores analizados presentan valores para todos los parámetros evaluados dentro de los límites considerados adecuados (Hair et al., 2009). Entonces, a partir de los resultados constantes de la Tabla 1, se consideró que el modelo de medición de los constructos del modelo de investigación se mostró en un grado apropiado para proseguir en el análisis de los resultados.

Seguidamente, se pasó a analizar el Modelo Estructural, donde se realizó inicialmente el Análisis Factorial Confirmatorio. Tomando como base los valores de referencia propuestos por Hair et al. (2009), se observa que los valores de los parámetros significancia, Qui-cuadrado $(1,774)$ normado y RMSEA $(0,061)$ se encuentran dentro de los límites establecidos como adecuados. Los parámetros TLI $(0,893)$ y CFI $(0,905)$ del modelo estructural a pesar de no atender los límites sugeridos por la bibliografía (Hair et al., 2009), se sitúan muy próximos a los límites sugeridos. Recordando aspectos como el hecho de que la muestra es relativamente pequeña para este modelo complejo (189 respuestas válidas) y que difícilmente una muestra de una encuesta cuantitativa empírica en empresas logra atender todos los parámetros estadísticos de acuerdo con los límites exigidos, se consideró que el modelo de investigación propuesto se mostraba válido de una forma mínimamente aceptable.

Pasando ahora al análisis de las relaciones entre los constructos del modelo estructural y la prueba de las hipótesis, la Tabla 2 presenta estos resultados.
En relación a las hipótesis relacionadas con la IORG sólo la relacionada con IPROD (H1a) fue rechazada, diferente de lo que Gunday (2011) demostró en las empresas de manufacturas en Turquía. Este resultado puede haber sido influenciado por la muestra de este estudio, pues la mayoría de las empresas son muy jóvenes y puede que no hayan tenido tiempo de innovaciones organizacionales que tengan efecto en las innovaciones de producto, pero fue de encuentro con los resultados obtenidos con enfoque en la industria de manufactura en Corea del Sur (Chung \& Kim, 2017).

Las innovaciones organizacionales mostraron una relación positiva con IPROC (H1b), demostrando que los cambios de estructura afectan directamente a los procesos organizacionales $\mathrm{c}$. La IORG también tiene una influencia positiva para IMKT (H1c), esto confirma que a medida que se realizan innovaciones en las empresas, esto afecta positivamente las innovaciones de marketing (Chung \& Kim, 2017; Gunday et al., 2011; Pino et al., 2016; Ulusoy et al., 2008)various papers investigate the effects of the technological (product and process.

En cuanto a la relación entre IPROC e IPROD (H2), la correlación fue positiva, con un resultado similar al que la literatura ya indica, reforzando la importancia de las innovaciones de proceso para las innovaciones de producto (Gunday et al., 2011; Ulusoy et al., 2008)various papers investigate the effects of the technological (product and process.

Tabla 2. Prueba de Hipótesis del modelo

\begin{tabular}{|c|c|c|c|c|c|c|c|c|}
\hline \multicolumn{4}{|c|}{ Hipótesis } & \multirow{2}{*}{$\begin{array}{c}\beta \\
, 024\end{array}$} & \multirow{2}{*}{$\begin{array}{c}\begin{array}{c}\text { Error } \\
\text { Estandarizado }\end{array} \\
, 024\end{array}$} & \multirow{2}{*}{$\begin{array}{l}\text { z-test } \\
\text {,200 }\end{array}$} & \multirow{2}{*}{$\begin{array}{c}\text { p-value } \\
, 841\end{array}$} & \multirow{2}{*}{$\begin{array}{l}\text { Prueba } \\
\text { Rechaza }\end{array}$} \\
\hline H1a & IPROD & $<--$ & IORG & & & & & \\
\hline $\mathrm{H} 1 \mathrm{~b}$ & IPROC & $<--$ & IORG & ,334 & ,334 & 3,865 & $* * *$ & Acepta \\
\hline $\mathrm{H} 1 \mathrm{c}$ & IMKT & $<--$ & IORG & ,751 & ,751 & 7,263 & $* * *$ & Acepta \\
\hline $\mathrm{H} 2$ & IPROD & $<--$ & IPROC & ,375 & ,375 & 2,831 & ,005 & Acepta \\
\hline H3a & IPROD & $<--$ & IMKT & 147 & 147 & 1,533 & 125 & Rechaza \\
\hline H3b & IPROC & $<--$ & IMKT & ,055 & ,055 & ,795 & ,426 & Rechaza \\
\hline $\mathrm{H} 4$ & DO & $<--$ & IPROD & ,036 & ,036 & ,397 & 691 & Rechaza \\
\hline H5 & DO & $<--$ & IPROC & ,623 & 623 & 4,305 & $* * *$ & Acepta \\
\hline $\mathrm{H} 6 \mathrm{c}$ & DO & $<--$ & IMKT & 167 & 167 & 2,084 & ,037 & Acepta \\
\hline
\end{tabular}

Fuente: Elaboración propia en base a los datos de la investigación. 
En lo que se refiere a las hipótesis que relacionan las innovaciones de marketing tanto relacionadas con IPROD (H3a) como IPROC (H3b) fueron rechazadas. Estos resultados son diferentes de los encontrados anteriormente a los relacionados a la IPROD (Gunday et al., 2011; Medrano \& Olarte-Pascual, 2016; Pino et al., 2016) (Gunday et al., 2011; Pino et al., 2016; Ulusoy et al., 2008)various papers investigate the effects of the technological (product and process e IMKT afectan positivamente las IPROC (Gunday et al., 2011; Medrano \& Olate-Pascual, 2016). Como las empresas son jóvenes, puede ocurrir que aún sean tímidos los resultados relacionados con la innovación de marketing, teniendo un impacto bajo en las innovaciones de producto y proceso.

La hipótesis que IPROD influencia el DO (H4) fue rechazada en desacuerdo con Gunday (2011) y Pino (2016), este resultado puede haber ocurrido, pues muchas innovaciones de producto necesitan un tiempo mayor para que los resultados sean observados en términos de desempeño organizacional (Madrid-Guijarro et al., 2013). Por último, las hipótesis que prueban IPROC e IMKT influenciando el DO (H5 y H6) fueron aceptadas, demostrando que el desempeño organizacional es afectado más rápidamente por las innovaciones de proceso y de marketing (Gunday et al., 2011; Pino et al., 2016).

\section{Conclusiones}

Buscando responder al problema de la investigación, el estudio trabajó el análisis de las relaciones de los tipos de innovación con el desempeño en parques tecnológico brasileños $y$, para ello, un modelo teórico fue creado y probado cuantitativamente (189 empresas), a través del modelaje de ecuaciones estructurales, donde se buscó analizar las relaciones propuesta.

Los resultados de los análisis estadísticos, permiten afirmar que existen interacción entre los tipos de innovación (Azar \& Ciabuschi, 2017; Gunday et al., 2011; Karabulut, 2015; Medrano \& Olate-Pascual, 2016; Pino et al., 2016; Ulusoy et al., 2008)various papers investigate the effects of the technological (product and process y de estas innovaciones con el desempeño de las empresas (Gunday et al., 2011; Madrid-Guijarro et al., 2013; Pino et al., 2016).

Incluso en las empresas que residen en un ecosistema de innovación, se encontraron resultados rechazados diferentemente de estudios hechos en otros ambientes como el de las empresas de manufactura en Turquía (Gunday et al., 2011), pequeñas y medianas empresas en España (Medrano \& Olate-Pascual, 2016), empresas exportadoras de las economías emergentes de América del Sur (Pino et al., 2016), en empresas de apoyo a la industria de mecánica, electrónica y automóvil en Hanoi, Vietnam (Tuan et al., 2016).

Como contribución teórica, este estudio ayudó a explorar el impacto de las innovaciones en las empresas, mostrando también que las innovaciones de producto necesitan un tiempo mayor para que se pueda visualizar sus resultados en términos de desempeño organizacional y lo mismo puede ser reportado de las innovaciones de marketing.

La investigación realizada también se muestra relevante, pues analizó la temática de los tipos de innovaciones y sus relaciones en el contexto de los parques tecnológicos en Brasil, trayendo insumos para que los gestores gubernamentales y de parques tecnológicos puedan utilizar en su toma de decisión.

Sin embargo, estos resultados deben ser visualizados a partir de las empresas residentes en parques tecnológicos brasileños, no pudiendo ser generalizados para contextos de muestras muy distintos de éste debido a las características peculiares brasileñas. Con el objetivo de superar esta limitación, se sugiere que nuevas colectas de datos sean realizadas con otras muestras, en otros contextos y en otros países y que puedan ser comparados los resultados en el futuro. 


\section{Referencias}

Aksoy, H. (2017). How do innovation culture, marketing innovation and product innovation affect the market performance of small and medium-sized enterprises (SMEs)? Technology in Society, 51, 133-141. https://doi. org/10.1016/j.techsoc.2017.08.005

Astuti, M., Prawoto, P., Irawan, Y. S., \& Sugiono, S. (2018). The eco-innovation variables which influence the performance of creative industries center of natural stone crafts. Journal of Ecological Engineering, 19(1), 1424. https://doi.org/10.12911/22998993/79446

Azar, G., \& Ciabuschi, F. (2017). Organizational innovation, technological innovation, and export performance: The effects of innovation radicalness and extensiveness. International Business Review, 26(2), 324-336. https:// doi.org/10.1016/j.ibusrev.2016.09.002

Chung, D. B., \& Kim, B. (2017). An analysis on the role of organizational innovation for enhancing firms' innovation performance focusing on the manufacturing industry in South Korea. In PICMET 2017 - Portland International Conference on Management of Engineering and Technology: Technology Management for the Interconnected World, Proceedings. https://doi.org/10.23919/PICMET.2017.8125366

Crossan, M. M., \& Apaydin, M. (2010). A multi-dimensional framework of organizational innovation: A systematic review of the literature. Journal of Management Studies, 47(6), 1154-1191. https://doi.org/10.1111/ j.1467-6486.2009.00880.x

Díeez-Vial, I., Fernández-Olmos, M., Díez-Vial, I., \& Fernández-Olmos, M. (2015). Knowledge spillovers in science and technology parks: how can firms benefit most? Journal of Technology Transfer, 40(1), 70-84. https://doi. org/10.1007/s10961-013-9329-4

Geldes, C., Felzensztein, C., \& Palacios-Fenech, J. (2017). Technological and non-technological innovations, performance and propensity to innovate across industriesThe case of an emerging economy. Industrial Marketing Management, 61, 55-66. https://doi.org/10.1016/j.indmarman.2016.10.010

Gkypali, A., Kokkinos, V., Bouras, C., \& Tsekouras, K. (2016). Science parks and regional innovation performance in fiscal austerity era: Less is more? Small Business Economics, 47(2), 313-330 . https://doi.org/10.1007/s11187016-9717-x

Gunday, G., Ulusoy, G., Kilic, K., \& Alpkan, L. (2011). Effects of innovation types on firm performance. International Journal of Production Economics, 133(2), 662-676. https://doi.org/10.1016/j.ijpe.2011.05.014

Hair, J. F., Black, W. C., Babin, B. J., Anderson, R. E., \& Tatham, R. L. (2009). Multivariate Data Analysis. Prentice Hall (7th ed). Upper Saddle River (NJ), USA: Prentice Hall.

Hassan, M. U., Shaukat, S., Nawaz, M. S., \& Naz, S. (2013). Effects of Innovation Types on Firm Performance: an Empirical Study on Pakistan's Manufacturing Sector. Pakistan Journal of Commerce and Social Sciences, 7(2), 243-262. http://www.jespk.net/publications/121.pdf

Hult, G. T. M., Hurley, R. F., \& Knight, G. A. (2004). Innovativeness: Its antecedents and impact on business performance. Industrial Marketing Management, 33(5), 429-438. https://doi.org/10.1016/j. indmarman.2003.08.015

Kahn, K. B. (2018). Understanding innovation. Business Horizons, 61(3), 453-460. https://doi.org/10.1016/j. bushor.2018.01.011

Karabulut, A. T. (2015). Effects of Innovation Types on Performance of Manufacturing Firms in Turkey. Procedia - Social and Behavioral Sciences, 195(3), 1355-1364 . https://doi.org/10.1016/j.sbspro.2015.06.322

Karlsson, C., \& Tavassoli, S. (2016). Innovation strategies of firms: What strategies and why? Journal of Technology Transfer, 41(6), 1483-1506. https://doi.org/10.1007/s10961-015-9453-4 
Madrid-Guijarro, A., García-Pérez-de-Lema, D., \& Van Auken, H. (2013). An investigation of spanish sme innovation during different economic conditions. Journal of Small Business Management, 51(4), 578-601. https://doi.org/10.1111/jsbm.12004

Medrano, N., \& Olarte-Pascual, C. (2016). The effects of the crisis on marketing innovation: an application for Spain. Journal of Business and Industrial Marketing, 31(3), 404-417. https://doi.org/10.1108/JBIM-02-2013-0048

OECD. (2007). Manual de Oslo: Diretrizes para a Coleta e Interpretação de dados sobre Inovação Tecnológica. OCDE, Eurostat e Financiadora de Estudos e Projetos, 3, 184. https://doi.org/10.1787/9789264065659-es

Pino, C., Felzensztein, C., Zwerg-villegas, A. M., \& Arias-bolzmann, L. (2016). Non-technological innovations : Market performance of exporting firms in South America. Journal of Business Research, 69(10), 4385-4393. https://doi.org/10.1016/j.jbusres.2016.03.061

Roldan, L. B. (2017). Condições favoráveis para a inovação em parques tecnológicos: Como estes ecossistemas de inovação influenciam a inovação das empresas e os seus resultados (1st ed.). Saarbrücken, Alemanha: Novas Edições Acadêmicas.

Schumpeter, J. A. (1939). Business cycles. NBER Books. New York, USA: McGraw-Hill.

Soetanto, D., \& Jack, S. (2016). The impact of university-based incubation support on the innovation strategy of academic spin-offs. Technovation, 50-51, 25-40. https://doi.org/10.1016/j.technovation.2015.11.001

Soetanto, D. P., \& Jack, S. L. (2013). Business incubators and the networks of technology-based firms. The Journal of Technology Transfer, 38(4), 432-453. https://doi.org/10.1007/s10961-011-9237-4

Tuan, N., Nhan, N., Giang, P., \& Ngoc, N. (2016). The effects of innovation on firm performance of supporting industries in Hanoi - Vietnam. Journal of Industrial Engineering and Management, 9(2), 413-431. https://doi. org/10.3926/jiem.1564

Ulusoy, G., Gunday, G., Alpkan, L., \& Kilic, K. (2008). Effects of organizational innovations on firms production performance. 2008 IEEE International Conference on Industrial Engineering and Engineering Management, 321325. https://doi.org/10.1109/IEEM.2008.4737883

Zuñiga-Collazos, A. (2018). Analysis of factors determining Colombia's tourist enterprises organizational innovations. Tourism and Hospitality Research, 18(2), 254-259. https://doi.org/10.1177/1467358416642008 